\title{
PRECEDENTES GRABADOS DEL ÉXTASIS DE LA BEATA LUDOVICA ALBERTONI DE BERNINI EN LA OBRA DE GUILLAUME VALET.
}

\author{
FERNANDO MORENO CUADRO \\ Universidad de Córdoba
}

\begin{abstract}
Resumen
El estudio presenta, dentro de las relaciones artísticas entre Italia y Francia durante el alto Barroco romano y el contexto de la difusión de la estampa del Carmelo teresiano, para el que trabajaron los más importantes maestros del momento, la vinculación del Éxtasis de la Beata Ludovica Albertoni de Bernini que preside la capilla Altieri de la iglesia de San Francesco a Ripa de Roma con el Éxtasis de santa Teresa transverberada grabado por Guillaume Valet para una de las grandes series dedicadas a la santa abulense durante el seiscientosPalabras clave
\end{abstract}

\section{Palabras clave}

Escultura, Barroco, iconografía, estampa.

\section{Abstract}

The study presents, within the artistic relations between Italy and France during the Roman High Baroque and the context of the diffusion of the Teresian Carmel design, which the most important maestros of the day were working on, the links between Bernini's "Ecstasy of Blessed Ludovica Albertoni" which hangs in the Altieri chapel of the Church of San Francesco a Ripa in Rome and the "Transverberation of Saint Teresa" etched by Guillaume Valet for one of the great series dedicated to the saint of Avila in the sixteenth century

$$
\text { Key Words }
$$

Sculpture, Baroque, iconography, design

\section{Introducción}

Muy abundantes han sido las aproximaciones a la obra de Gianlorenzo Bernini (1598-1680), uno de los grandes genios del alto Barroco romano, al que dedicamos estas notas incidiendo en su aportación y vinculación a la iconografía teresiana, recordando el Éxtasis de Santa Teresa (1647-1652) de Santa Maria della Vittoria de Roma y presentando los antecedentes grabados de la Vita Effigiata et Essercizi Affettivi di S. Teresa di Giesv Maestra di Celeste Doctrina (Roma, Mascardi, 1670) para una de sus más prestigiosas realizaciones, el Éxtasis de la beata 


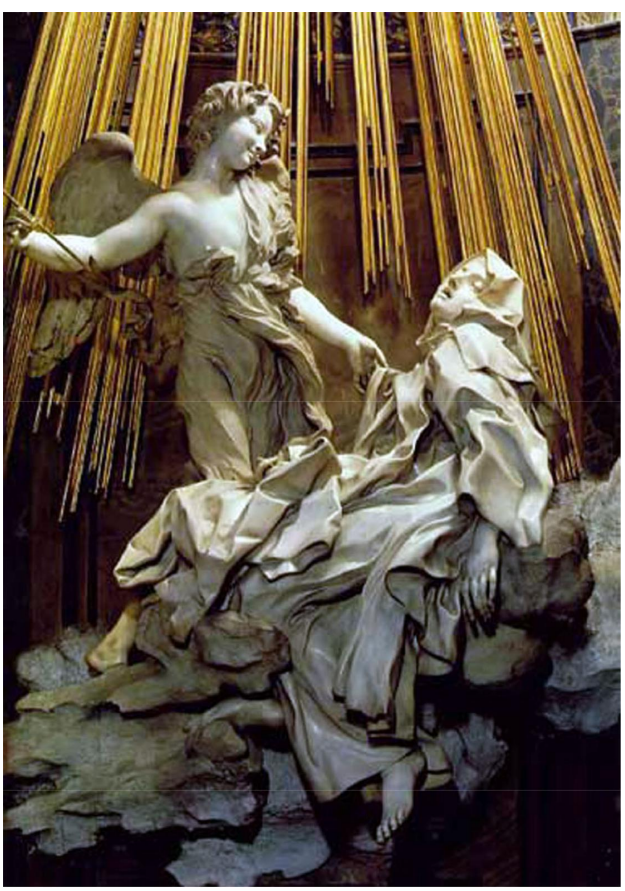

Ludovica Albertoni (1671-1674) que preside
Fig 1. Gian Lorenzo Bernini, Éxtasis de santa Teresa, Capilla Cornaro, Santa María de la Victoria de Roma, 1647-1652

la capilla Altieri de la iglesia romana de San Francesco a Ripa ${ }^{1}$.

Un cuarto de siglo separan las más importantes esculturas berninescas que ma-terializan los éxtasis de la reformadora del Carmelo y de la terciaria franciscana ${ }^{2}$. La experiencia teresiana de la transverberación ${ }^{3}$ ha tenido una gran trascendencia artística ${ }^{4}$, desde versiones populares a obras contemporáneas ${ }^{5}$, siendo uno de los máximos exponentes el grupo de la capilla Cornaro ${ }^{6}$, el ejemplar más perfecto de las relaciones de color en la obra del artista napolitano, en el que destaca la blancura del grupo que acentúa el dramatismo de la escena y la teatralidad del conjunto, pre-sentando la transverberación de santa Teresa sobre una nube suspendida, iluminada por un foco de luz amarillenta -especialmente efectista al atardecer, subrayando lo efímero del momento representado- con sus connotaciones simbólicas, fundamen-talmente dirigida a la persona que recibe la Gracia divina, que introduce a los fieles -entre los que se han destacado, en los palcos laterales, siete cardenales y un duque de la familia Cornaro6, obra de los mejores discípulos de Bernini ${ }^{7}$ - en la experiencia sobrenatural que contemplan, trasladando a la importante capilla, concebida como un enorme lienzo, la ilusión interpretada por los pintores ${ }^{8}$.

Sería prácticamente imposible, y sobrepasa los límites propuestos, acercarnos a la influencia del excepcional grupo de Bernini, pues su impronta aparece en numerosas obras, no solo de

${ }^{1}$ Véase Sheely Karen Perlove, Bernini and the idealization of Death: The Blessed Ludovico Albertoni and the Altieri Chapel. Pennsylvania State University Press, 1990

2 Véase Ferrini-Ramírez, Santos franciscanos. Asís, Editorial Porciúncula, 2000, p. 35. 687

3 J. Chevalier: "Sainte Thérèse d'Ávila et l'expérience de Dieu", en Histoire de la Pensé, Paris, 1956. pp. 669-

4 W. Weibel, “The Representation of Ecstasy”, en Bernini in Perspective. New Jersey, 1976.

5 . Saitajoki, "Pyhän Teresam Hurmio", en Acta Universitatis Lapponiensis, 61 (2003), 110 pp.

${ }^{6}$ R. Kuhn: "Die Unio Mystica der Hl. Therese von Avila von Lorenzo Bernini in der Kornaro Kapelle in Rom”, en Alte un Moderne Kunst, XII (1967), pp. 2-8.

7 Santa María de la Victoria, Roma, ATS Italia Editrice, 1999. p. 15

${ }^{8}$ R. Wittkower: Arte y arquitectura en Italia, 1660-1750. Manuales Arte Cátedra. Madrid, 1979. pp. 157-160. 
colecciones particulares $^{9}$ y museos -pequeño cobre $(30$ x $22 \mathrm{~cm}$.) de Escuela Española de la segunda mitad del siglo XVII conservado en el Museo de Bellas Artes de Granada ${ }^{10}$, , sino también en pinturas callejeras -decoración de la fachada de la casa $\mathrm{n}^{\circ} .1$ de la Kirchplatz de la localidad austriaca de Natters, enel Tirol- y de conventos, como el monasterio del Carmen de San Rafael o Carmen Bajo de Santiago Chile ${ }^{11}$, por citar algunas obras muy diversas.

En muchos casos se reproduce fielmente el modelo berninesco y en otros se adapta, como en la estampa de L. Valperga sobre dibujo de Giovanni Comandí, en la que se reduce la representación al busto de santa Teresa que aparece bajo el Espíritu Santo sosteniendo el dardo de Amor divino, quebrado como un rayo, con la mano izquierda, al tiempo que sostiene con la derecha el hábito que en la escultura de Benini aparta el ángel. Es un tipo de transposición habitual, queriendo mostrar origi-nalidad sin perder la deuda con el gran maestro, cuya vinculación con la iconografía teresiana trasciende la del famoso grupo de la Transverberación comentado, siendo evidente el paralelismo de la escultura de la Beata Ludovica Albertoni con una de las escasas estampas firmadas por Guillaume Valet (1632-1704) para la Vita... citada de 1670, que se completa con el texto "B. Teresia diuino amore aestuans, et aureo, / ignitoq(ue) spiculo pluries: transuerberata / seraphicas inter acies elanguet" (La beata Teresa $^{12}$, ardiendo de amor divino y traspasada varias veces por un dardo de fuego, se desmaya entre legiones de serafines) ${ }^{13}$, que se recoge también en la Vie de la Mere Sainte Teresa de Iesus..., publicada el mismo año en Lyon ${ }^{14}$, y fue seguida por la estampa suelta -Teresianum de Roma-, lo que subraya el éxito de la composición. La originalidad de la escultura de la iglesia trastiberina no radica solo en el excepcional uso del color característico de Bernini ${ }^{15}$, sino también en la composición. No conocemos otros éxtasis - "transformamiento del alma del todo en Dios (que) dura poco" Bernini completa el grupo a los lados del lienzo de Santa Ana Triplex del Baciccia (c. 1675) que sirve de fondo y preside el retablo de la capilla Altieri ${ }^{17}$, cuyas miradas repiten las de los ángeles grabados por Guillaume Valet, incluyen-do los que más pendientes de la luz celeste o del espectador se muestran ajenos al personaje que circundan ${ }^{18}$. Ambas religiosas están recostadas con las piernas flex-ionadas, iluminadas por una fuente de luz oculta, aunque la contenida conquista del cuerpo por el ropaje y el gozo espiritual del rostro en la excepcional

9 Alcalá Subastas. Subasta 35, lote 67.

10 Museo de Bellas Artes de Granada. Inventario CE0419.

11 A. Rojas Abrigo, Historia de la pintura en Chile. Santiago, Banco Español Chile, 1981, p. 204; L. Mebold, Catálogo de pintura colonial en Chile, Universidad Católica de Chile, 1987, p. 374.

12 El término <beatus $>$ (bienaventurado) se aplica por la Iglesia Católica a quienes en el camino de la santificación han superado los estadios de $<$ Siervo de Dios $>$ y $<$ Venerable $>$, considerando que han tenido una vida de santidad y esperan el proceso de canonización que la declare como modelo de conducta a la Iglesia Universal. No se usa el término < sanctus $>$ hasta que no se ha terminado el proceso de canonización con la solemne declaración papal de que una persona está contemplando la visión de Dios y, en cambio, si se utiliza el término < beatus> (dichoso, por gozar del paraíso) aplicado a santos, como en la estampa grabada por Guillaume Valet que comentamos

13 Vita Effigiata et Essercizi Affettivi di s. Teresa di Giesv Maestra di Celeste Doctrina, Roma, 1670, p. 264.

${ }^{14} \mathrm{La}$ Vie de la seraphique Mere Sainte Teresa de Iesvs, fondatrice des Carmes Déchaussez \& Carmelites Déchaussées, en Figuras, \& en Vers Francois \& Latins. Avec un Abbregé de l'Histoire, une Reflexion Morale, \& une Resolution Chrétienne sur châque Figura, Lyon, 1670, ilus. 18, p. 117.

${ }^{15}$ R. Wittkower: Arte y arquitectura en Italia, 1660-1750. Manuales Arte Cátedra. Madrid, 1979. pp. 155-157.

${ }^{16}$ Santa Teresa de Jesús, Vida, 20, 18

17 Roma e dintorni. Milano, Guida d'Italia del Touring Club Italiano, 1977, p. 468

18 En los grupos enmarcados por los triángulos con vértices en los rostros de las extáticas, encontramos ángeles con la mirada no centrada en las protagonistas, mientras que la mayoría observa fijamente su rostro, como podemos observar también en el grupo de ángeles circunscrito en el triángulo con base paralela a los cuerpos de Teresa y Ludovica, cuyos rostros contemplan, excepto los que en la Capilla Altieri pierden la mirada en la luz divina, como alguno de la estampa de Valet, en la que destaca especialmente el que llama la atención del espectador para que centre su atención en la santa abulense 
escultura de la terciaria franciscana superan la estampa, en la que la santa de Ávila presenta una mano sobre el vientre y otra extendida, mientras que Ludovica Albertoni las aprieta sobre el pecho y vientre, como Valet, aunque con más intensidad que en la estampa del grabador francés. En todo caso, además de las vinculaciones señaladas, lo importante es mostrar el arrobamiento del que habla tan frecuentemente la santa en sus escritos, y tanto la escultura del Trastevere como la estampa citada, son de los ejemplos más importantes del alto barroco que muestran el éxtasis místico, del que habló san Francisco de Sales, siguiendo muy de cerca a santa Teresa ${ }^{19}$, quien en Las Moradas trata especialmente del arrobamiento ${ }^{20}$, sobre el que también escribe en su Vida

\begin{abstract}
"Digo que muchas veces me parecía me dejaba el cuerpo tan ligero, que toda la pesadumbre de él me quitaba, y algunas era tanto, que casi no entendía poner los pies en el suelo. Pues cuando está en el arrobamiento, el cuerpo queda como muerto, sin poder nada de sí muchas veces, y como le toma se queda: si en pie, si sentado, si las manos abiertas, si cerradas. Porque aunque pocas veces se pierde el sentido, algunas me ha acaecido a mí perderle del todo, pocas y poco rato. Mas lo ordinario es que se turba y aunque no puede hacer nada de sí cuanto a lo exterior, no deja de entender y oír como cosa de lejos. No digo que entiende y oye cuando está en lo subido de él (digo subido, en los tiem-pos que se pierden las potencias, porque están muy unidas con Dios), que entonces no ve ni oye ni siente, a mi parecer; mas, como dije en la oración de unión pasada, este transformamiento del alma del todo en Dios dura poco; mas eso que dura, ninguna potencia se siente, ni sabe lo que pasa allí. No debe ser para que se entienda mientras vivimos en la tierra, al menos no lo quiere Dios, que no debemos ser capaces para ello. Yo esto he visto por mín"21.
\end{abstract}

Poco sabemos de la obra de Guillaume Valet, salvo que grabó fundamentalmente asuntos de historia y religiosos, como las estampas de la Vita... comentada, y fue discípulo de Carlo Maratta (1625-1713) ${ }^{22}$, cuya obra se caracterizó por un estilo sobrio y mesurado y el manejo fácil del lenguaje alegórico del momento ${ }^{23}$, habi endo trabajado en sus años juveniles como grabador, antes de la década de 1660, en la que se intensifica la actividad artística en la corte francesa ${ }^{24}$ y las relaciones entre París y Roma. En 1666 Luis XIV fundó la Académie de France à Roma, bajo la dirección de Jean-Baptiste Colbert, Charles Le Brun y Gianlorenzo Bernini, que el año anterior había sido llamado a París ${ }^{25}$, donde realizó su tercer proyecto para el Louvre ${ }^{26}$. La misma década en que François Girardon visita Roma para refrescar los

19 San Francisco de Sales, Tratado del Amor de Dios, Madrid, BAC, 1995, libros VI, VII y VIII, 19 Alcalá Subastas. Subasta 35, lote 67

${ }^{20}$ Santa Teresa de Jesús, Las Moradas. Moradas Sextas, cap. 4, en que “Trata de cuando suspende Dios el alma en oración con arrobamiento o éxtasis o rapto, que todo es uno a mi parecer, y cómo es menester gran ánimo para recibir tan grandes mercedes de su Majestad

${ }^{21}$ Santa Teresa de Jesús, Vida, 20, 18

22 E. Bénézit: Dictionnaire critique et documentaire ede peintres, sculteurs, desinateurs et graveurs. París, 1924, t. X, p. 461, le adjudica el monograma "VF enlazadas" que corresponde a su hijo Jérome Vallet (1667-1702), véase al respecto F. Bruillet, Dictionnaire des monogrammes, marques, figurées, lettres initials, noms, abrégés, etc. avec lesquels les peintres, dessinateurs, graveurs et sculpteurs ont désigné leurs noms. Munich, L'Institut Literaire Artistique de la Librairie de J. G. Cotta, 1832.

23 O. Kunstchera-Woborsky, "Ein Kunsttheoretisches Thesenblatt des Carlo Maratti", en Graphische Künste, Mitteilungen (1919), p. 9. Cfr. R. . Wittkower: Arte y arquitectura en Italia, 1660-1750. op. cit. p. 337

24 A. Blunt, Arte y Arquitectura en Francia 1500/1700. Madrid, Manuales Arte Cátedra, 1977, pp. 331 ss

25 Véase P. Freart de Chanteloup, Diario del viaje a Francia del Caballero Bernini. Murcia, Colegio de Aparejadores y Arquitectos, 1986

${ }^{26}$ E. Esmonin, "Le Bernin et la construction du Louvre", en B.S.H.A.F. (1911), p. 31 
Precedentes grabados del Éxtasis de la Beata Ludovica Albertoni
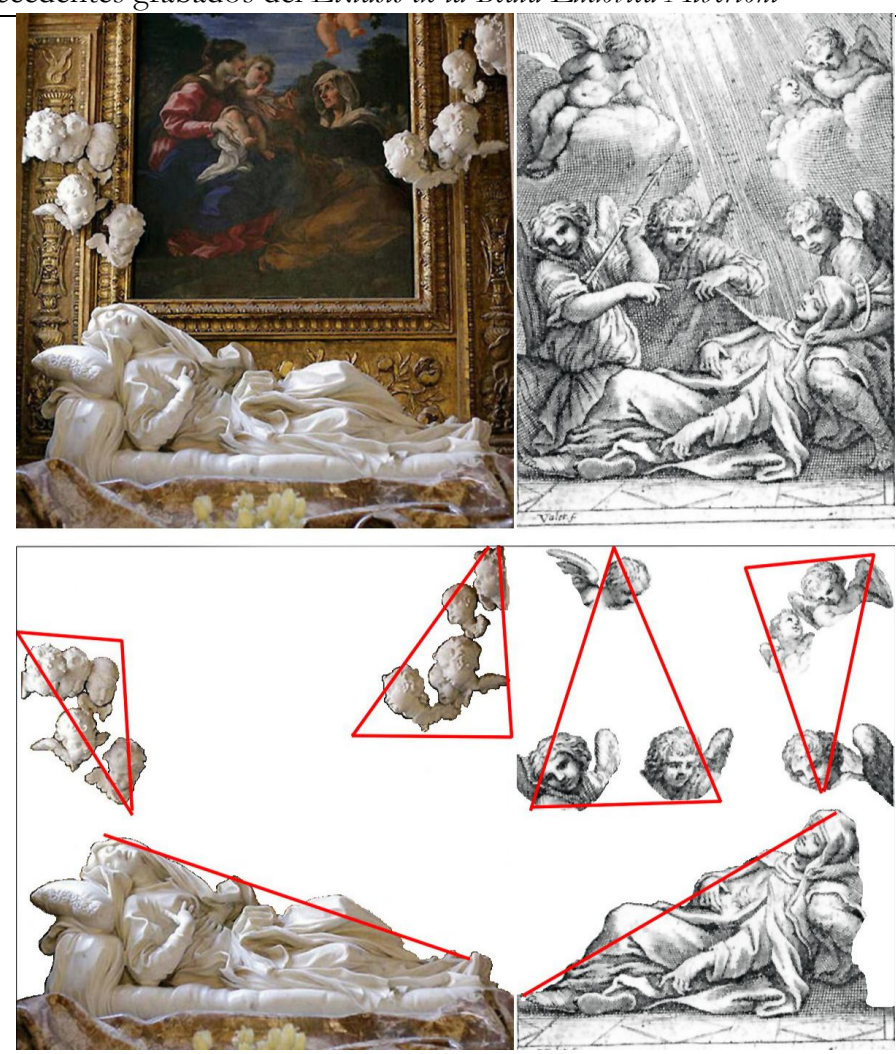

Fig 2. Gian Lorenzo Bernini, Éxtasis de la beata Ludovica Albertoni, Capilla Al-tieri, San Francesco a Ripa de Roma, 1671-1674, y Guillaume Valet, Éxtasis de santa Teresa transverberada, de la Vita Effigiata... Roma, 1670 (arriba) y esquemas con los principales elementos compositivos de ambas obras, coincidentes en la dis-tribución y compensación del peso y dirección de las formas (abajo).

recuerdos de la escultura clásica que utiliza en la decoración de Versalles ${ }^{27}$, copiando años más tarde una figura de Nicolás Poussin, que pasó toda su vida activa en Roma ${ }^{28}$, para la tumba de Richelieu (1675-1677) en la iglesia de la Sorbona de Paris, con el cuerpo del cardenal reclinado sobre el sarcófago, asistido por la figura alegórica de la Piedad ${ }^{29}$ que sigue la mirada del clérigo francés que fundó la Academia francesa -en la que ingresó Guillaume Valet el 19 de julio de 1664-, cuyo director Le Brun pintó para la iglesia carmelitana del arrabal de Saint-Jacques de París un cuadro representando el Saint Esprit apparaissant à Sainte Thérèse d'Avila el día de Pentecostés, después de asistir a misa, cuando se retiró al lugar donde solía rezar y meditar con el apoyo de lecturas ${ }^{30}$, conocido a través de Mariette ${ }^{31}$,

\footnotetext{
27 P. Francastel, Girardon. 1928.

28 A. Blunt, Nicolas Poussin. Londres, 1967-1968

${ }^{29}$ R. A. Weigert, "Deux marchés inédites pour la tombe de Richelieu", en Bulletin de la Societé Poussin, I (1947), p. 67

30 Santa Teresa de Jesús, Vida, 38.

31 D. Wildenstein, "Les oeuvres de Charles Le Brun d'après les gravures de son temps”, en Gažette des BeauxArts, julio-agosto, 1965 , pp. 1-58
} 
quien grabó el modelo carmelitano que -a diferencia del realizado por Rubens ${ }^{32}$, en el que aparece la santa arrodillada observando a la tercera Persona de la Trinidad, con la quietud que siguió al sobresalto ${ }^{33}$ - se asocia a la transverberación en varios grabados y pinturas ${ }^{34}$.

Las relaciones entre Francia e Italia también se hacen patentes en uno de los hitos fundamentales de iconografía teresiana en el seiscientos, la aparición de las series de 1670 que replantean la iconografía de la santa abulense surgida en la Vita S. Virginis Teresiae a Iesvs, que fue publicada en Amberes, en casa de Adrianum Collardum y Cornelius Galleum, en 1613 -cuando se inicia el proceso de beatificación- y reeditada en 1630 por el éxito que tuvo, no sintiéndose la necesidad de realizar una nueva serie teresiana más amplia hasta 1670, cuando se editarán la Vita effigiata... y Le Vie... simultáneamente en Roma y Lyon, después de difundirse la Vita iconibus expresa (1669) dedicada a santa María Magdalena de Pazzi, cuya canonización se celebró el 28 de abril de 1669 en la Basílica de San Pedro de Roma junto a la del franciscano san Pedro de Alcántara, lo que originó una rica y variada iconografía alcantarina ${ }^{35}$ y de la extática carmelita calzada ${ }^{36}$. Pensamos que éste fue el detonante para replantear la iconografía teresiana, ya que había pasado medio siglo desde que se realizó en Flandes la serie relativamente pequeña -veinticuatro planchas- dedicada a santa Teresa en 1613 y con motivo de su canonización en 1622, al estar muy cercana la primera, no se planteó la realización de una nueva serie y sólo se insistió especialmente en uno de los temas fundamentales de su iconografía: la transverberación, que fue el motivo central de la estampa oficial $^{37}$. Estamos firmemente convencidos de que los nuevos tiempos y especialmente el amplio desarrollo que comienzan a tener las series dedicadas a otros santos en esta época son los condicionantes del planteamiento y realización de las grandes series dedicadas a santa Teresa en el segundo tercio del siglo XVII que están en la base del lienzo Giovanni Battista Tiépolo, María exhortando a santa Teresa para que nombrara a san José protector de la Orden, del Szépmüvészeti Múzeum de Budapest, más directamente relacionado con la adaptación de las

32 Cfr. L. Gutiérrez Rueda: "Ensayo de iconografía teresiana”, en Revista de Espiritualidad, 90 (1964), p. 66.

${ }^{33}$ Sobre la vinculación de Rubens con los Carmelitas véase F. Moreno Cuadro, "Rubens y el Carmelo. Entre la estampa de invención y la de reproducción”, en Boletín del Museo e Instituto Camón Aznar, 106 (2010).

${ }^{34}$ Muy semejantes son las estampas de devoción -en el pie de una de ellas se lee "S. Mater Teresa ora / pro nobis"- en las que desde el Espíritu Santo surge el dardo encendido con una llama que tiene la forma de corazón al que se dirige. Sin apenas cambios en el espacio en el ejemplar de la Curia Generalicia de O.C.D. de Roma, mientras que en la estampa del Instituto

Histórico del Teresianum de Roma se ha insertado un crucificado en la pared ante el que se sitúa el reclinatorio. Se trata de un tipo de estampa muy particular que representa un modelo que con ligeras variantes tuvo influencia en otras estampas y un cierto desarrollo en pinturas, entre las que destacan las conservadas en la Curia Generalicia de O.C.D. de Roma y en la Colección Carmelitana de Francia (L'Art du XVIIe siècle dans les Carmels de France. Catálogo de la exposición celebrada en el Musée du Petit Palais, 17 de nov. 1982 - 15 febr. 1983, París, ediciones Yves Rocher, 1982, p. 48, n. 8) que añade un crucifijo, como la citada estampa del Teresianum, al tiempo que amplia la ventana y coloca bajo ella algunos de los atributos característicos de la santa, libro, flagelo y calavera. Mayor abertura al exterior se observa en la versión conservada en el monasterio de MM.CC.DD. de México, donde se completa la singular visión teresiana que se realiza en un espacio abierto a un jardín con un pequeño angelito que le dispara el dardo, adaptándose a la tradición, como se hace más evidente en el ejemplar del Carmelo de Clamart (L'Art du XVIIe siècle dans les Carmels de France... p. 53, n. 13) y en la estampa de F. Hiller con la que guarda relación un singular lienzo de colección particular (Alcalá Subastas. Miércoles, 15 de diciembre de 1999. Subasta 1, lote 49)

35 S. Andrés Ordax: Arte e iconografía de san Pedro de Alcántara. Institución Gran Duque de Alba. Diputación Provincial de Ávila, 2002.

36 F. Moreno Cuadro, "Iconografía de Magdalena de Pazzi. A propósito de Alonso Cano, Valdés Leal y Pedro de Moya", en Locus Amoenus, 10, 2009-2010, pp. 141-152 p. 99

37 Publicada por E. Mâle: L'art religieux de la fin du XVIe siècle, du XVIIe siècle et du XVIIIe siècle. Paris, 1932, 
mismas a la estética del setecientos en la Vita Effigiata Della Serafica Vergine S. Teresa di Gesù fondatrice dell'Ordine Carmelita Scalro, grabada de Arnol van Westerhout en $1715^{38}$.

Finalmente, comentar que hablamos de series porque ilustran diferentes obras -Vita ... y Vie... de 1670-, pero realmente es una serie que presenta leves difer-encias. Ambas se editan simultáneamente en dos de los más importantes centros productivos de estampas en Europa -Lyon y Roma- ilustrando las obras citadas. El hecho de que algunos grabados estén firmados por Gillaume Valet y que se de-staque la devoción de Santa Teresa a San Luis ${ }^{39}$, advierten del origen francés de la serie, aunque ésta debió realizarse probablemente en Roma, pues la serie italiana es mayor, es la que presenta tres estampas firmadas por Valet y en ella se advierte el recuerdo de Giovanni Lanfranco, el discípulo de Agostino Carracci que a princip ios de los años 30 realizó para el monasterio de San José de MM.C.D. una pintura de Santa Teresa recibiendo el collar de manos de María en presencia de San José, que fue seguida por Valet en una estampa de la serie italiana que firma y aparece reproducida en espejo respecto al lienzo de Lanfranco y a la serie francesa, por lo que podríamos considerarla como la estampa de la plancha original al invertir el modelo, si bien pudo haber utilizado alguna estampa de reproducción de la singular pintura, de la que conocemos el ejemplar conservado en el Colegio Internacional San Alberto O.C. de Roma. Las variantes de las dos series son mínimas, cuando no se repiten exactamente igual, presentándose algo más de dos tercios invertidas en las ediciones citadas. Pequeñas diferencias que se deben a que dado que la edición es simultánea, con lo que cuenta el país importador es con las pruebas de estado, de editor o autor que se hacían para comprobar el efecto general de la estampa, que se puede cambiar si no es satisfactorio, a lo que pensamos se deben los cambios señalados, que no aparecen en la estampa en que nos centramos y consideramos el precedente grabado utilizado por Bernini al plantear el Extasis de la Beata Ludovi-ca Albertoni de la capilla Altieri de la iglesia romana de San Francesco a Ripa.

38 F. Moreno Cuadro, "En torno a las fuentes iconográficas de Tiépolo para la $<$ Visión teresiana $>$ del Museo de Bellas Artes de Budapest", en Archivo Español de Arte, LXXXIV, 32, 2009, pp. 243-258.

39 A san Luis se atribuye la presencia de los carmelitas en Occidente, siendo especialmente interesante en este sentido el vitral de Boxmeer (C. Emond: L'Iconographie Carmélitaine dans les anciens Pays-Bas méridionaux. Bruselas, 1961. p. 201.) que siguiendo la estampa de Clouwet para Les peintures sacrees du Temple du Carmel (Liége, 1669) -ilustrado con veintiséis estampas de los más importantes grabadores flamencos del momento dibujadas por Abraham Diepenbeke (1596-1675)- rep-resenta a cuatro carmelitas que van a embarcar acompañando al rey que fundó el primer convento de carmelitas en París (J. B. Lezana: Annales sacri et prophetici et Eliani ordinis Beatis. Virginis Mariae de Monte Carmeli. Roma, 1645-1656, 4. vols. V. IV, pp. 470-471), aunque la verdadera razón de la llegada a Occidente está en la inseguridad de Tierra Santa a principios del siglo XIII a pesar de los pactos entre cruzados y sarracenos. Vicente de Beauvais concreta la fecha en 1238 (Biblioteca mundi, IV, Douai, 1624, p. 1275), pero el abandono definitivo sería a finales de la centuria cuando los cruzados fueron final-mente vencidos por los sarracenos. 